\title{
Endocrine and Metabolic Disorders in Children With Cancer Treatment
}

\author{
Maryam Nakhaei-Moghadam, ${ }^{1,}$ Aria Setoodeh, ${ }^{2}$ Noormohammad Noori, ${ }^{1}$ Parastoo Rostami, ${ }^{2}$ and \\ Alireza Teimouri ${ }^{1}$ \\ ${ }^{1}$ Children and Adolescent Health Research Center, Zahedan University of Medical Sciences, Zahedan, IR Iran \\ ${ }^{2}$ Department of Pediatrics, Faculty of Medicine, Tehran University of Medical Sciences, Tehran, IR Iran \\ "Corresponding author: Maryam Nakhaei-Moghadam, Children and Adolescent Health Research Center, Zahedan University of Medical Sciences, Zahedan, IR Iran. E-mail: \\ maryamnakhaey@yahoo.com
}

Received 2015 April 23; Accepted 2015 November 16.

\begin{abstract}
Background: Survivors of childhood malignancy are at risk of long-term late effects. One of the most commonly involved systems in this effect is endocrine system. Appropriate timely recognition and treatment of late complications including endocrine complication is essential for the continuing health and improvement in quality of life in cancer survivors.

Objectives: Therefore, this study has been conducted to investigate the aforementioned complications resulting from the treatment of the common malignancies in children in Iran.

Patients and Methods: We performed a cross sectional study for evaluation of endocrine complication in cancer survivors who had finished their cancer treatment and come for followed up in oncology clinic of children medical center.

Results: Demographic data, treatment modality, and endocrine late effect recorded and analyzed. There were 96 survivors with median age of $13.8 \pm 5.8$ years (range 3 - 31 years). The median follow up time was 2.7 years (range 1 - 10 years). Hyperinsulinemia was the most common late effect. Other complication was dyslipidemia, overweight, obesity, osteopenia, hypothyroidism, diabetes mellitus and osteoporosis respectively.

Conclusions: Our study has demonstrated a significant prevalence of endocrine complication after childhood cancer therapy and a long term follow-up program for survivors of childhood cancer is therefore needed.
\end{abstract}

Keywords: Malignancy, Childhood, Endocrine Complication, Metabolic Complication

\section{Background}

Almost one out of every thousand children before the age of 15 years is suffering from cancer. The most common malignancies in children are acute leukemia (32\%), Hodgkin lymphoma (10\%) and brain tumors (24\%) [1]. One of the most remarkable progresses in medical sciences over the past four decades is an effective treatment for pediatric malignancies and this will prolong the life of these patients to survive more. Survival rate of 5 years from less than $30 \%$ in 1960 to over $70 \%$ was reached in 1990 . This rate is more than $90 \%$ for some malignancies such as leukemia and lymphoma [2]. Currently, one out of every 640 persons aged 20 - 39 years is a survivors of childhood cancer and in the near future this amount will be one in every 450 persons [3] and about $60 \%$ of them have at least one chronic condition due to malignant [4].

However, using the treatment of cancer at early age can cause effects that may not become apparent until many years later which are expressed late effects. This term means the adverse effects which is associated with the treatment on various organs and psychological dis- orders and experienced by the patients after cessation of treatment and these adverse effects including endocrine disorders, heart and lung, liver, kidney, secondary malignancy, psychosocial problems and even premature death [1]. Childhood cancer survivors have an increased risk of endocrine disease affecting many organs: hypothalamicpituitary function, gonadal and reproductive function thyroid function, body composition and glucose homeostasis [5].

Endocrine complications that caused by radiotherapy and chemotherapy would be directly due to endocrine function decline or caused by gland tissue metaplasia indirectly due to the incidence of malignancy and disorder in bone growth [6]. Sensitivity to the toxic effects of treatment in endocrine glands varies (depending on the characteristics of each tumor cell). Some endocrine organs such as the testes, which have a high rate of cell division is most sensitive to the effects of anti-neoplastic drug and radiation [5]. Among other endocrine abnormalities may point to dysfunction of the hypothalamus, pituitary hypothyroidism, diabetes insipidus and mellitus, impaired growth hormone and thyroid disorders $[1,6]$. With these 
given findings, a history of cancer and its treatment will have a significant and long-term effect on health of population at risk. Over time, these populations are less likely to visit their primary centers to follow-up treatment, often are not aware of their previous treatment and the majority of them have not received screening programs based on any previous treatment.

Not more debates over whether the children should have long-term follow-up. In many centers of cancer treatment in the world, long-term follow-up is existed to evaluate long term complications of the disease and provide services to these patients according to pre-screening and evaluation of these effects [7]. In the last 20 years, there have been considerable improvements in the treatment protocols for childhood cancer so that an effective cure rate can now be obtained with substantially fewer late effects [8].

\section{Objectives}

Appropriate and on time recognition and treatment its complications, improved quality of life for these children. Therefore, this study has been conducted to investigate the aforementioned complications resulting from the treatment of the common malignancies in children in Iran.

\section{Patients and Methods}

This cross-sectional study operated on previously treated cases of childhood cancer in the oncology department at children's medical center in 2011, which were referred to a hematologist for follow-up. Random number tabulation was used to collect 96 children and those with simultaneously diseases that affect the endocrine system were excluded. After obtaining written consent from patients or their families, primary and basic information including the type of cancer, age, type of treatment (chemotherapy, radiation and surgery), therapeutic doses, age at start of treatment and the treatment history, collected from the oncology ward and medical record of hospital. radiation dose achieved from patients of the cancer institute of Imam Khomeini hospital cases had obtained the necessary coordination with the center had been done to it. Patients who were in needing of radiotherapy referred to the institute of Imam Khomeini hospital from children's center cancer. Clinical examinations were performed on patients including; assessment of vital signs, growth parameters (height measured using a stadiometer and weight using scales). These measures were reviewed on a growth chart, Tanner stage classification system for puberty and other routine physical examinations. All obtained information from medical records, physical examination and laboratory findings were recorded in a specific survey form. For the analyzing data SPSS-13 was used and statistical $\chi^{2}$, independent $t$ and multiple regressions tests. The significant level considered of $\mathrm{P}<0.05$.

\section{Results}

Out of 96 patients, $42.7 \%$ were girls. The mean age at the time of study was 13.8 with a range of 3 - 31 years and the mean age at the time of the primary diagnosis was 5.4 with 1-14 years range. The duration of treatment was varied from 1 to 10 years with a median of 2.7 years. The time after stopping treatment had a range of 1-25 years with the average of 6.1 years. Most primary malignancy was for leukemia with 64 patients (66.7\%) and followed with lymphoma of 15 patients (15.6\%), brain tumors with 7 patients (7.3\%) and other kinds of tumors (rhabdomyosarcoma, Wilms, neuroblastoma, Ewing's sarcoma) with 10 patients (10.4\%) were ranked in the third and fourth orders.

According to the type of cancer, 40 patients (40.6\%) received chemotherapy, 37 patients (37.3\%) received simultaneous treatments of chemotherapy and radiation therapy, and 3 patients $(2.1 \%)$ received treatments with surgery and radiotherapy. Chemotherapy was doing with different combinations included, vincristine, methotrexate, cyclophosphamide, cytozar, prednisolone, asparginase mercaptopurine and doxorubicin. Unfortunately, prescribed dose and cumulative dose were not able to achieve from medical records. Out of 96 patients, 56 had radiotherapy. For these patients, the total dose of radiation $(27.5 \pm 2.18$ Gy) with range of 12 - $68 \mathrm{~Gy}$, the fractionation dose and each fractioned dose were $15.1 \pm 1.4$ Gy with range of 4 - 60 Gy and $1.9 \pm 0.7$ Gy with range of 1.5 - 4.5 Gy respectively. Patients with height below the fifth percentile were $18.8 \%$. Diagnosis of acute leukemia was the greatest. These patients were treated with chemotherapy alone or combined with radiotherapy. The analysis resulted that, the heights of $33 \%$ of patients were within 5 - 10th percentiles and $20 \%$ were between 10 - 25 percentiles but significant association not existed between short stature and type of treatment. Based on the measures of BMI, 28 patients (29.2\%) were overweight and 16 patients (16.7\%) were obese in which significant association not observed between weight gain or obesity and type of treatment, duration of therapy, sex or age of the patients and time interval after stopping treatments.

Hyperinsulinemia in 44 patients (45.8\%), type 2 diabetes in 2 patients (2.1\%), vitamin D deficit in 76 (79.1\%) and hyperlipidemia in 19 patients (19.8\%) was observed. From patients with hyperlipidemia, 11 (57.8\%) and 8 (42.1\%) 
patients had gain in triglycerides and cholesterol respectively based on age and sex. Not observed any association between any of the listed disorders with treatment, weight, gender and time since last treatment. Puberty had developed in $70(1.70 \%)$ patients at the appropriate time and 23 patients were in the age before maturity. Delayed in puberty or early puberty was observed.

Table 1. The Frequency and Percentage Distribution of Endocrine Complications in Childhood Cancer Survivors

\begin{tabular}{|lc|}
\hline Endocrine Disorder & No. (\%) \\
\hline Vitamin D deficiency & $79.1(76)$ \\
\hline Hyperinsulinemia & $45.8(44)$ \\
\hline Weight gain & $29.2(28)$ \\
\hline Dyslipidemia & $19.8(19)$ \\
\hline Short stature & $18.7(18)$ \\
\hline Overweight $\left(\mathbf{B M I}>\mathbf{2 5} \mathbf{k g} / \mathbf{m}^{\mathbf{2}}\right)$ & $16.7(16)$ \\
\hline Hypothyroidism & $8.3(8)$ \\
\hline Underweight $\left(\mathbf{B M I}<\mathbf{1 8} \mathbf{~} \mathbf{g g} / \mathbf{m}^{\mathbf{2}}\right)$ & $6.3(6)$ \\
\hline Type 2 diabetes & $2.1(2)$ \\
\hline
\end{tabular}

Primary hypothyroidism in $8(3.8 \%)$ patients was observed in which 7 were boys and 3 were diagnosed with subclinical hypothyroidism. From these patients, 62\% with Hodgkin lymphoma in neck and neck radiotherapy were done. The average radiation dose was 27.7 Gy and registered. In this study, none of the patients had central hypothyroidism (Table 1). In addition, among patients in study, dysfunction of hypothalamic-pituitary axis, adrenal system, gonads and parathyroid not observed at all.

\section{Discussion}

In this study out of these patients, $57.3 \%$ were boys. The mean age at the time of study was 13.8 years with range of 3 - 31 years. The mean age at the time of the primary diagnosis was 5.4 years with 1 - 14 years range. The treatment duration ranged from 1 to 10 years with a median of 2.7 years. The time after stopping treatment had a range of $1-25$ years with the average of 6.1 years. Most primary malignancy was for leukemia (66.7\%) and followed with lymphoma (15.6\%), brain tumors (7.3\%) and 10.4\% for other kinds of tumors. Endocrine disorder is due to dysfunction (increase or reduction) in any of the endocrine gland. These disorders can occur as primary or secondary to other conditions. One of the secondary endocrine disorders is malignancies [1, 9-11]. In a study conducted by Skowronska-Gardas et al. observed that $40 \%$ of 650 patients survived from childhood cancer had endocrine problems and other complications were in the prevalence rate of visual-auditory disorders-cognitive dysfunction, heart-lung and gastrointestinal and $30 \%$ of patients had no problem [3]. The results conducted by Skowronska-Gardas et al. are comparable with our results when we resulted that, the percentage for hyperinsulinemia was $45.8 \%$. In previous studies reported that $10 \%-15 \%$ of leukemia survivors had height below of $5 \%$ percentile when in the present study the rate of $18 \%$ was achieved in cancer survivors [1]. In a study has been carried out by Armenian et al. 57.6\% of patients with a history of malignancy childhood at least had one endocrine disorder caused by cancer treatment when $22 \%$ had multilump involvement [6]. The most common problems associated with weight disorders (decrease or increase) observed in $31 \%$ of patients, gonadal dysfunction in $25.2 \%$ of patients and $19.4 \%$ had impaired growth [8] in which these results is similar with our findings.

Reduction in height growth is a common finding in childhood cancer survivors. The causes of reduced growth are including of growth hormone deficiency, hypothyroidism, radiation therapy, corticosteroid and use of flat skull beads [6]. In previous studies, $10 \%-15 \%$ of survivors of leukemia have been reported with the height lower than fifth percentile when in the present study $18 \%$ for this percentage was found. It is recommended that, to be measured the growth parameters every 6 months till full growth and then every year [7]. Wasilewski-Masker in the own study reported that $31 \%$ of patients with leukemia had BMI > 30 and an increasing of hypercholesterolemia and hypertriglyceridemia 13 years after diagnosis. These amounts in population who had radiotherapy compared to those who received only chemotherapy significantly increased [12].

Meanwhile, based on our study $29.2 \%$ was overweight and $16.7 \%$ was obese. Also a significant correlation between overweight and obesity was not found in which was based on treatment, duration of therapy, sex and age of the patients and the treatment interval. It was different from the findings of previous research.

In a sense, our results in terms of hypercholesterolemia and hypertriglyceridemia were similar to previous research.

Young adult Hodgkin disease survivors who were treated with high doses of radiation to the thyroid gland are at substantially increased risk for the development of a spectrum of abnormalities of the thyroid. Female survivors are at particularly high risk for developing hypothyroidism and thyroid nodules [5, 8]. According to the records the most common thyroid disorder due to the cancer treatment is hypothyroidism and this occurs in average after 2 to 7 years $[4,12,13]$. In various reviews the in- 
cidence of $2-50 \%$ has been reported for hypothyroidism [14] which is similar with our findings. The relationship between radiation and hyperparathyroidism was reported in 1975. Latent period between radiation and remarkable hyperparathyroidism in clinical aspect has been reported of 35 - 49 years [15]. In our study not found any cases of parathyroid dysfunction.

According to the findings of Darzy and Shalet study less than $5 \%$ of children with cancer are affected to hyperprolactinemia after radiotherapy [16]. In our study, hyperprolactinemia and other functional disorders of hypothalamic pituitary axis was not observed.

The prevalence of vitamin D deficiency is common and high in cancer survivors and is similar with the general population. The importance role of vitamin D deficiency is being a cause of cardiovascular disease, the incidence of malignancy and bone loss in patients with cancer in which is likely these problems are higher in patients with malignancy [5]. Early recognition and treatment can reduce morbidity and mortality in this vulnerable population. The importance of long-term surveillance of those at risk cannot be over emphasized. These endocrine abnormalities may evolve over many years. One of the future challenges will be to better characterize the role of genetic variability in the pathogenesis of these endocrine abnormalities. According to the findings of current and previous studies long term investigation on patients who suffering from cancers but stopped the treatment is recommended regularly and routinely. Therefore, screening them in terms of growth, maturation processes and semen analysis, evaluation of thyroid and parathyroid function tests, measurement of cortisol, prolactin and vitamin D levels in blood, assessment of BMI, fasting blood glucose, insulin and lipid profile, at specified intervals after cancer treatment is recommended.

\section{Acknowledgments}

This study is a part of fellowship thesis done by $\mathrm{Dr}$ Maryam Nakhaei-Moghadam.

\section{Footnote}

Authors' Contribution: All authors participated equally in the following tasks: conception and design, data collection, statistical analysis and interpretation, writing with critical revision, final approval and overall responsibility.

\section{References}

1. Landier W, Armenian S, Bhatia S. Late effects of childhood cancer and its treatment. Pediatr Clin North Am. 2015;62(1):275-300. doi: 10.1016/j.pcl.2014.09.017. [PubMed: 25435123].

2. Shad A, Myers SN, Hennessy K. Late effects in cancer survivors: "the shared care model". Curr Oncol Rep. 2012;14(2):182-90. doi: 10.1007/s11912-012-0224-1. [PubMed: 22286374].

3. Skowronska-Gardas A, Pedziwiatr K, Chojnacka M. Evaluation of quality of life in long-term survivors of paediatric brain stem tumors, treated with radiotherapy. Radiother Oncol. 2004;70(3):269-73. doi: 10.1016/j.radonc.2004.01.008. [PubMed:15064012].

4. Hancock SL, Cox RS, McDougall IR. Thyroid diseases after treatment of Hodgkin's disease. N Engl J Med. 1991;325(9):599-605. doi 10.1056/NEJM199108293250902. [PubMed:1861693]

5. Chemaitilly W, Sklar CA. Endocrine complications in long-term survivors of childhood cancers. Endocr Relat Cancer. 2010;17(3):R141-59. doi: 10.1677/ERC-10-0002. [PubMed: 20453080].

6. Armenian SH, Robison LL. Childhood cancer survivorship: an update on evolving paradigms for understanding pathogenesis and screening for therapy-related late effects. Curr Opin Pediatr. 2013;25(1):16-22. doi:10.1097/MOP.ob013e32835bob6a. [PubMed: 23295717].

7. Nandagopal R, Laverdiere C, Mulrooney D, Hudson MM, Meacham L. Endocrine late effects of childhood cancer therapy: a report from the Children's Oncology Group. Horm Res. 2008;69(2):65-74. doi: 10.1159/000111809. [PubMed: 18059086].

8. Brignardello E, Felicetti F, Castiglione A, Chiabotto P, Corrias A, Fagioli $\mathrm{F}$, et al. Endocrine health conditions in adult survivors of childhood cancer: the need for specialized adult-focused follow-up clinics. Eur J Endocrinol. 2013;168(3):465-72. doi: 10.1530/EJE-12-1043. [PubMed 23258270].

9. van Santen HM, Thonissen NM, de Kraker J, Vulsma T. Changes in thyroid hormone state in children receiving chemotherapy. Clin Endocrinol (Oxf). 2005;62(2):250-7. doi: 10.1111/j.1365-2265.2005.02210.x. [PubMed: 15670204].

10. Spoudeas HA. Growth and endocrine function after chemotherapy and radiotherapy in childhood. Eur J Cancer. 2002;38(13):1748-59. [PubMed: 12175691].

11. Oberfield SE, Allen JC, Pollack J, New MI, Levine LS. Long-term endocrine sequelae after treatment of medulloblastoma: prospective study of growth and thyroid function. J Pediatr. 1986;108(2):219-23. [PubMed: 3944706].

12. Wasilewski-Masker K, Kaste SC, Hudson MM, Esiashvili N, Mattano LA Meacham LR. Bone mineral density deficits in survivors of childhood cancer: long-term follow-up guidelines and review of the literature. Pediatrics. 2008;121(3):e705-13. doi: 10.1542/peds.2007-1396. [PubMed: 18310191].

13. Massimino M, Gandola L, Seregni E, Bongarzone I, Morosi C, Collini P Thyroid iatrogenic sequelae after the treatment of pediatric cancer. $Q$ J Nucl Med Mol Imaging. 2009;53(5):526-35.

14. Murray RD. Endocrine management of the cancer survivor. 12th ed. Philadelphia: W.B. Saunders; 2011. pp. 1782-808.

15. Fulbright JM, Raman S, McClellan WS, August KJ. Late effects of childhood leukemia therapy. Curr Hematol MaligRep. 2011;6(3):195-205. doi: 10.1007/s11899-011-0094-x. [PubMed: 21695425].

16. Darzy KH, Shalet SM. Hypopituitarism following radiotherapy. Pituitary. 2009;12(1):40-50. doi: 10.1007/s11102-008-0088-4. [PubMed: $18270844]$. 